\title{
Nonlinear Motion Detection ${ }^{\star}$
}

\author{
Lennart Wietzke and Gerald Sommer
}

Cognitive Systems Group

Kiel University, Department of Computer Science

Christian-Albrechts-Platz 4, D-24118 Kiel, Germany

lw@ks.informatik.uni-kiel.de

\begin{abstract}
This work presents new ideas in multidimensional signal theory: an isotropic quadrature filter approach for extracting local features of arbitrary curved signals without the use of any steering techniques. We unify scale space, local amplitude, orientation, phase and curvature in one framework. The main idea is to lift up signals by a conformal mapping to the higher dimensional conformal space where the local signal features can be analyzed with more degrees of freedom compared to the flat space of the original signal domain. The philosophy is based on the idea to make use of the relation of the conformal signal to geometric entities such as hyper-planes and hyper-spheres. Furthermore, the conformal signal can not only be applied to $2 \mathrm{D}$ and $3 \mathrm{D}$ signals but also to signals of any dimension. The main advantages in practical applications are the rotational invariance, the low computational time complexity, the easy implementation into existing Computer Vision software packages, and the numerical robustness of calculating exact local curvature of signals without the need of any derivatives. Applications can be optical flow and object tracking not only limited to constant velocities but detecting also arbitrary accelerations which correspond to the local curvature.
\end{abstract}

\section{Introduction}

Low level image analysis is often the first step of many Computer Vision tasks. Therefore, local signal features determine the quality of subsequent higher level processing steps. It is important not to lose or to merge any of the original information within the local neighborhood of the point of interest. The constraints of local signal analysis are: to span an orthogonal feature space (split of identity) and to be robust against stochastic and deterministic deviations between the actual signal and the model. Image signals $f \in L^{2}(\Omega)$ with $\Omega \subset \mathbb{R}^{n}$ will be locally analyzed on a low level. The assumed local signal model is defined as a hyper-sphere

$$
\mathcal{P}\{f\}(z ; s)=(f * p)(z ; s)=a \cos \left(\left\|z-\frac{1}{\kappa} \bar{o}\right\|+\phi\right), \quad(z, s) \in \Omega \times \mathbb{R}_{+}
$$

with $a$ as the local amplitude, $\phi \in[0, \pi)$ as the local phase, $\kappa>0$ as the local curvature and $\bar{o}$ as the normal of the local orientation. For the special case of

\footnotetext{
* We acknowledge funding by the German Research Foundation (DFG) under the project $\mathrm{SO} 320 / 4-2$.
}

X. Jiang and N. Petkov (Eds.): CAIP 2009, LNCS 5702, pp. $11221129,2009$.
(C) Springer-Verlag Berlin Heidelberg 2009 


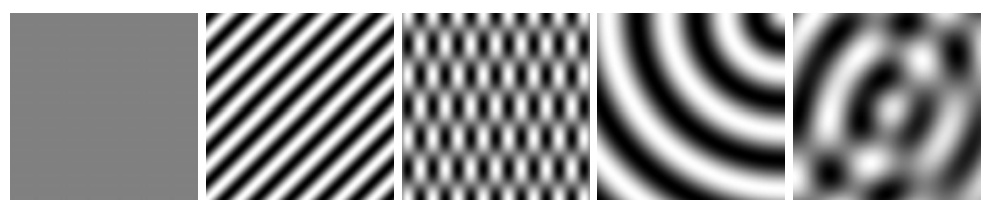

Fig. 1. From left to right: a constant signal (i0D), an arbitrary rotated 1D signal (i1D) and an $\mathrm{i} 2 \mathrm{D}$ checkerboard signal consisting of two simple superimposed i1D signals. A curved i2D signal and two superimposed curved i2D signals. Note that all signals displayed here preserve their intrinsic dimension globally.

$\kappa=0$ the hyper-sphere degrades to a hyper-plane. One important local structural feature is the phase $\phi$ which can be calculated by means of the Hilbert transform [6]. Furthermore all signals will be analyzed in Poisson scale space $\mathcal{P}\{\cdot\}$ [3] since the Hilbert transform can only be interpreted for narrow banded signals. The Poisson kernel of the applied low pass filter reads

$$
p_{n}(z ; s)=\frac{2}{A_{n+1}} \frac{s}{\left(s^{2}+\|z\|^{2}\right)^{(n+1) / 2}}, \quad A_{n+1}=\frac{2 \pi^{\frac{n+1}{2}}}{\Gamma\left(\frac{n+1}{2}\right)}
$$

with $A_{n+1}$ as the surface area of the unit sphere $\mathbb{S}^{n}\left[10\right.$ in Euclidian space $\mathbb{R}^{n+1}$.

\section{Related Work}

Local phase and amplitude of 1D signals can be analyzed by the analytic signal 6]. The generalization of the analytic signal to multidimensional signal domains has been done by the monogenic signal [2]. The $n D$ monogenic signal is restricted to i1D signals for all dimensions. The monogenic signal replaces the classical onedimensional Hilbert transform of the analytic signal by the generalized Hilbert transform [1] in Euclidean space

$$
h_{n}(z)=\frac{2}{A_{n+1}} \frac{z}{\|z\|^{n+1}}, \quad z \in \mathbb{R}^{n}, n \in \mathbb{N} \backslash\{1\}
$$

This paper shows, that signal analysis problems can be solved in higher dimensional conformal spaces 911. Since the original signal will be analyzed in scale space it will be of advantage to summarize the Hilbert kernel and the Poisson to one unified kernel which will be called the conjugated kernel

$$
q_{n}(z ; s)=\left(h_{n} * p_{n}\right)(z ; s)=\frac{2}{A_{n+1}} \frac{z}{\left(s^{2}+\|z\|^{2}\right)^{(n+1) / 2}}
$$

To enable interpretation of the generalized Hilbert transform, its relation to the Radon transform is the key [12 of signal intelligence, see Figures (4) and (3). The generalized Hilbert transform can be expressed by a concatenation of the Radon transform, the inverse Radon transform and the well known classical 1D Hilbert transform kernel $h(t)=\frac{1}{\pi t}$. Note that the relation to the Radon transform is required solely for interpretation and theoretical results. Neither the 

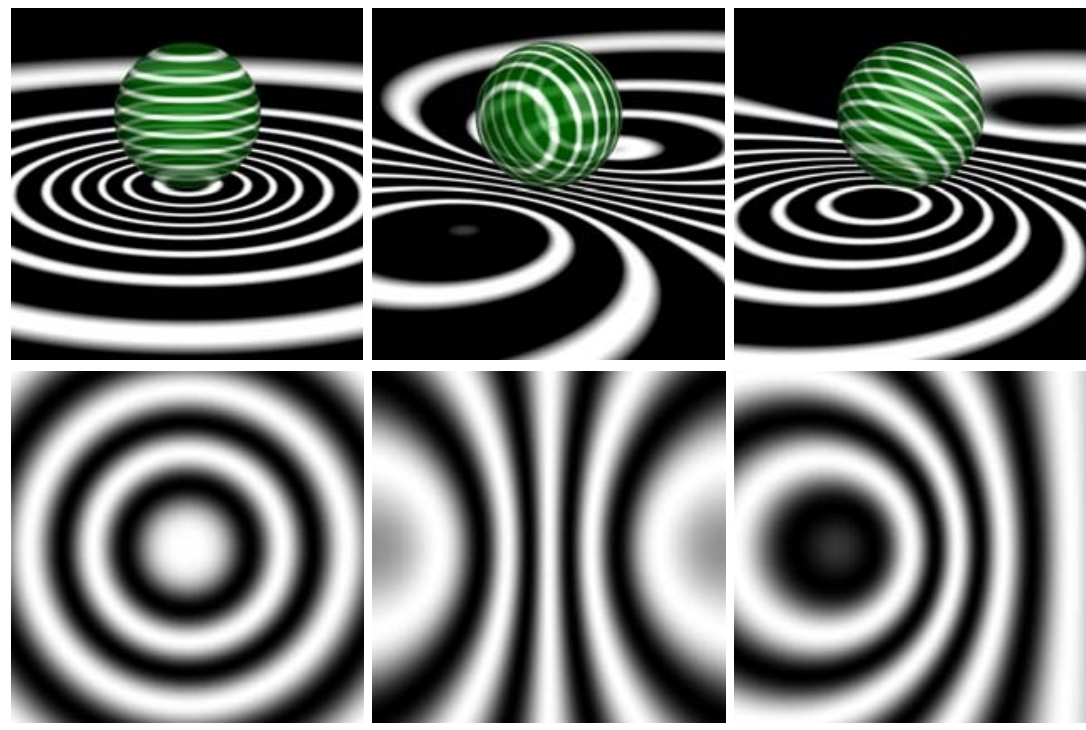

Fig. 2. Illustration of the conformal mapping of $2 \mathrm{D}$ signals to the $3 \mathrm{D}$ conformal space

Radon transform nor its inverse are ever applied to the signal in practice. Instead the generalized Hilbert transformed signal will be determined by convolution in spatial domain.

\section{The Conformal Signal}

In case of visual motion analysis a three dimensional isotropic quadrature filters are needed $7 / 4$. The conformal signal of a $3 \mathrm{D}$ signal $f \in L^{2}(\Omega)$ with $\Omega \subset \mathbb{R}^{3}$ delivers amplitude, $3 \mathrm{D}$ orientation, phase and curvature. For image sequences (3D signals) the concept of planes in 3D Radon space becomes the more abstract concept of hyper-planes in 4D Radon space. These 4D hyper-planes determine $3 \mathrm{D}$ spheres on the $4 \mathrm{D}$ hyper-sphere in $4 \mathrm{D}$ conformal space. Since 3D planes and $3 \mathrm{D}$ spheres of the three-dimensional signal domain are mapped to 3D spheres on the $4 \mathrm{D}$ hyper-sphere, the integration on these 3D spheres determines points in the 4D Radon space. The general inverse stereographic projection for any dimension $n \in \mathbb{N}$ which maps the Euclidian space $\mathbb{R}^{n}$ to the conformal space $\mathbb{R}^{n+1}$ reads

$$
\mathcal{S}^{-1}\left(x_{1}, x_{2}, \ldots, x_{n}\right)=\frac{1}{1+\sum_{\nu=1}^{n} x_{\nu}^{2}}\left[\begin{array}{c}
x_{1} \\
x_{2} \\
\vdots \\
x_{n} \\
\sum_{\nu=1}^{n} x_{\nu}^{2}
\end{array}\right]
$$

The inverse stereographic projection maps the Euclidian space $\mathbb{R}^{n}$ to the hypersphere in $\mathbb{R}^{n+1}$ with radius $\frac{1}{2}$ and the south pole of the hyper-sphere touching 
the origin $\mathbf{0} \in \mathbb{R}^{n}$ of the Euclidian space $\mathbb{R}^{n}$ and the north pole of the hypersphere with coordinates $(\mathbf{0}, 1) \in \mathbb{R}^{n+1}$. For the signal dimension $n=3$ the inverse stereographic projection $\mathcal{S}^{-1}$ known from complex analysis [5] maps the $3 \mathrm{D}$ signal domain to the hyper-sphere. This projection is conformal and can be inverted by the general formula

$$
\mathcal{S}\left(\xi_{1}, \xi_{2}, \ldots, \xi_{n}, \xi_{n+1}\right)=\frac{1}{1-\xi_{n+1}}\left[\begin{array}{c}
\xi_{1} \\
\xi_{2} \\
\vdots \\
\xi_{n}
\end{array}\right]
$$

The back-projection $\mathcal{S}$ for all elements of the hyper-sphere reads with $\xi=$ $\left(\xi_{1}, \xi_{2}, \xi_{3}, \xi_{4}\right)$. This mapping has the property that the origin of the 3D signal domain will be mapped to the south pole $\mathbf{0}$ of the hyper-sphere and both $-\infty,+\infty$ will be mapped to the north pole $(0,0,0,1)$ of the hyper-sphere. $3 \mathrm{D}$ planes and spheres of the 3D signal domain will be mapped to spheres on the hyper-sphere and can be determined uniquely by hyper-planes in 4D Radon space. The integration on these hyper-planes corresponds to points $\left(t, \theta_{1}, \theta_{2}, \varphi\right)$ in the $4 \mathrm{D}$ Radon space. Since the signal domain $\Omega \subset \mathbb{R}^{3}$ is bounded, not the whole hyper-sphere is covered by the original signal. Anyway, all hyper-planes corresponding to spheres on the hyper-sphere remain unchanged. That is the reason why the conformal signal models 3D planes and all kinds of curved 3D planes which can be locally approximated by spheres. To provide the generalized Hilbert transform more degrees of freedom, the original three-dimensional signal will be embedded in an applicable subspace of the conformal space by the so called conformal signal $c \in \mathbb{R}^{\left(\mathbb{R}^{4}\right)}$ of the original $3 \mathrm{D}$ signal $f$

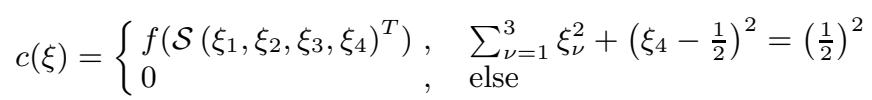

by which the even signal part $c^{e}=\left(c * p_{4}\right)(\mathbf{0} ; s)$ can by defined. Thus, the 4D generalized Hilbert transform can be applied to all points on the hyper-sphere. The center of convolution in spatial domain is the south pole where the origin of the 3D signal domain meets the hyper-sphere. At this point the generalized Hilbert transform will be evaluated in spatial domain by convolution for each test point

$$
c^{o}=\left[\begin{array}{c}
c_{1}^{o} \\
c_{2}^{o} \\
c_{3}^{o} \\
c_{4}^{o}
\end{array}\right]=\left(q_{4} * c\right)(\mathbf{0} ; s)=\frac{2}{A_{5}} \int_{z \in \mathbb{R}^{4}} \frac{z}{\left(s^{2}+\|x\|^{2}\right)^{5 / 2}} c(z-\mathbf{0}) d z
$$

The conformal signal for 3D signals is defined by the even part and the four odd parts of the 4D Hilbert transform. Note that the coordinates are relative to the local coordinate system for each test point of the original 3D signal and $\mathbf{0}=(0,0,0,0)$ are the corresponding relative coordinates in conformal space, i.e. this is no restriction. The Hilbert transform of the $3 \mathrm{D}$ signal embedded in the conformal space can also be written in terms of the 4D Radon transform and its inverse 

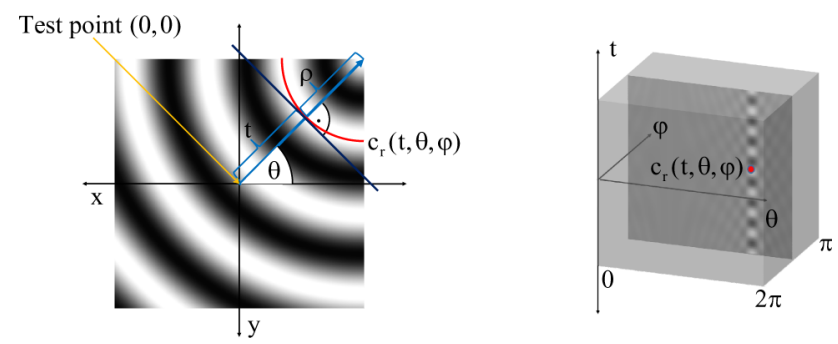

Fig. 3. Left figure: Curved i2D signal with orientation $\theta$ and curvature $\kappa=\frac{1}{\rho}$. Right figure: Corresponding 3D Radon space representation of the $\mathrm{i} 2 \mathrm{D}$ signal spanned by the parameters $t, \theta$ and $\varphi$. Since the Radon transform on circles directly on the plane of the original 2D signal is not invertible, the Radon transform has to be done in higher dimensional 3D conformal space where circles correspond to planes.

$$
c^{o}=\mathcal{R}^{-1}\left\{\left[\begin{array}{r}
\cos \varphi \sin \theta_{1} \sin \theta_{2} \\
\sin \varphi \sin \theta_{1} \sin \theta_{2} \\
\cos \theta_{1} \sin \theta_{2} \\
\cos \theta_{2}
\end{array}\right] h(t) * \mathcal{R}\left\{\left(c * p_{4}\right)(z ; s)\right\}\left(t ; \theta_{1}, \theta_{2}, \varphi\right)\right\}(\mathbf{0} ; s)
$$

This representation of the Hilbert transform is essential for the subsequent interpretation of the conformal signal. Remember that without loss of generality the signal will be analyzed at the origin of the local coordinate system of the test point of local interest. Compared to the monogenic signal the conformal signal is based on a Hilbert transformation in conformal space. Analogous to the interpretation of the monogenic signal in [12, the parameters of the hyperplane within the 4D Radon space determine the local features of the curved 3D signal. The conformal signal can be called the generalized monogenic signal for $3 \mathrm{D}$ signals, because the special case of planes in the original $3 \mathrm{D}$ signal can be considered as spheres with zero curvature. These planes are mapped to spheres passing through the north pole in conformal space. The $3 \mathrm{D}$ curvature corresponds to the parameter $\varphi$ of the $4 \mathrm{D}$ Radon space,

$$
\varphi=\arctan \frac{c_{2}^{o}}{c_{1}^{o}}
$$

Besides, the curvature of the conformal signal naturally indicates the intrinsic dimension of the signal. The parameters $\left(\theta_{1}, \theta_{2}\right)$ will be interpreted as the orientation of the signal in the original $3 \mathrm{D}$ space

$$
\theta_{1}=\arcsin \frac{\sqrt{\left[c_{1}^{o}\right]^{2}+\left[c_{2}^{o}\right]^{2}}}{c_{4}^{o}} \text { and } \theta_{2}=\arctan \frac{\sqrt{\left[c_{1}^{o}\right]^{2}+\left[c_{2}^{o}\right]^{2}+\left[c_{3}^{o}\right]^{2}}}{c_{4}^{o}}
$$

The amplitude and phase are defined by

$$
a=\sqrt{\left[c^{e}\right]^{2}+\left\|c^{o}\right\|^{2}} \text { and } \phi=\arctan \frac{\left\|c^{o}\right\|}{c^{e}}
$$

In all different intrinsic dimensions the phase indicates a measure of parity symmetry. Note that all proofs are analogous to those for the monogenic signal shown in 12 . 


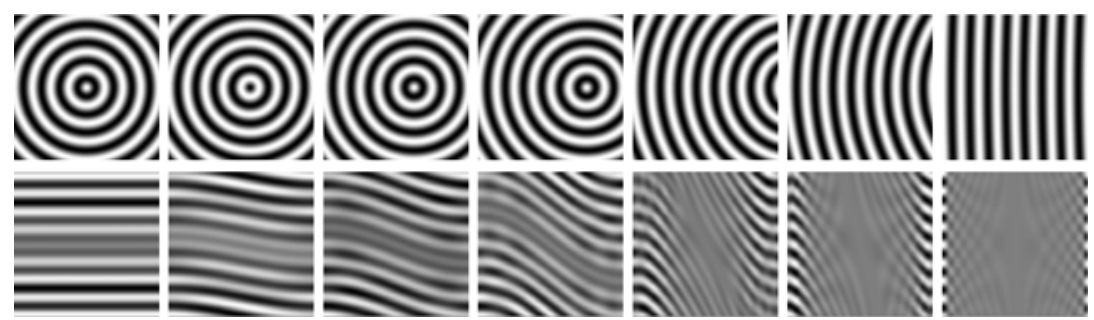

Fig. 4. From left to right: signal with varying curvature in spatial domain (top row) and in the corresponding 2D Radon space (bottom row). Obviously the 2D Radon space is too flat for analyzing and parameterizing the orientation and curvature of signals. Therefore the dimension of the $2 \mathrm{D}$ Radon space must be extended to 3D. This is the idea of the conformal signal.

\subsection{Implementation}

The computational time complexity is in $O\left(n^{3}\right)$ with $n$ as the convolution mask size in one dimension.

//Input: double Image3D(double $x$,double y,double $z$ )

//Input: double $\mathrm{x}, \mathrm{y}, \mathrm{z}$ (Local pixel test point for analysis)

//Input: double Coarse > Fine > 0 (Bandpass filter parameters)

//Input: double Size > 0 (Convolution mask size)

//Output: Direction1, Direction2, Phase, Curvature, Amplitude

double Coarse=2,Fine=0.1; int Size=5;//e.g .

double $r p=0, r 1=0, r 2=0, r 3=0, r 4=0$;

for (double cx = -Size; $c x<=$ Size; $c x+=1$ )

for (double cy $=-$ Size; cy < Size; cy $+=1$ )

for (double cz $=-$ Size; $c z<=$ Size; $c z+=1$ )

\{

//Map points (cx, cy, cz) to conformal space ( $1, x 2, x 3, x 4)$

double $d=$ pow $(c x, 2)+$ pow $(c y, 2)+$ pow $(c z, 2)+1$;

double $\mathrm{x} 1=\mathrm{cx} \quad / \mathrm{d}$;

double $\mathrm{x} 2=\mathrm{cy} \quad / \mathrm{d}$;

double $\mathrm{x} 3=\mathrm{cz} / \mathrm{d}$;

double $\mathrm{x} 4=(\mathrm{d}-1) / \mathrm{d}$;

$/ /$ Generalized Hilbert transform in conformal space

double $\mathrm{a}=\operatorname{pow}(\mathrm{x} 1,2)+$ pow $(\mathrm{x} 2,2)+$ pow $(x 3,2)+$ pow $(\mathrm{x} 4,2)$;

double pf $=$ pow $($ pow $($ Fine, 2$)+a,-2.5)$;

double pc $=$ pow $($ pow $($ Coarse, 2$)+a,-2.5)$;

double $f=$ Image $3 D(x+c x, y+c y, z+c z)$;

double $\mathrm{c}=\mathrm{f} *(\mathrm{pf}-\mathrm{pc})$;

$\mathrm{rp}+=\mathrm{f} *$ (Fine*pf - Coarse*pc);

\}

$\mathrm{r} 1+=\mathrm{x} 1 * \mathrm{c} ; \mathrm{r} 2+=\mathrm{x} 2 * \mathrm{c} ; \mathrm{r} 3+=\mathrm{x} 3 * \mathrm{c} ; \mathrm{r} 4+=\mathrm{x} 4 * \mathrm{c}$;

Curvature $=\operatorname{atan}(r 2 / r 1)$;

Direction1 $=\operatorname{asin}(\operatorname{sqrt}(\operatorname{pow}(r 1,2)+\operatorname{pow}(r 2,2)) / r 4)$; 


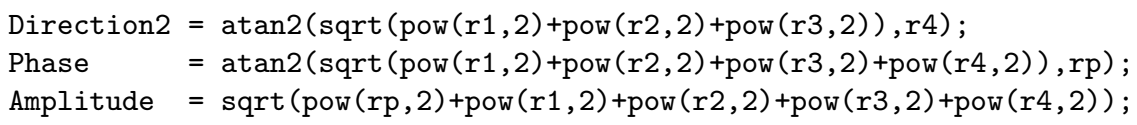

In practical applications such as medical image analysis [4] or 3D optical flow (5) the convolution mask sizes must be DC-free. This can be achieved by removing their mean value after precalculating them.
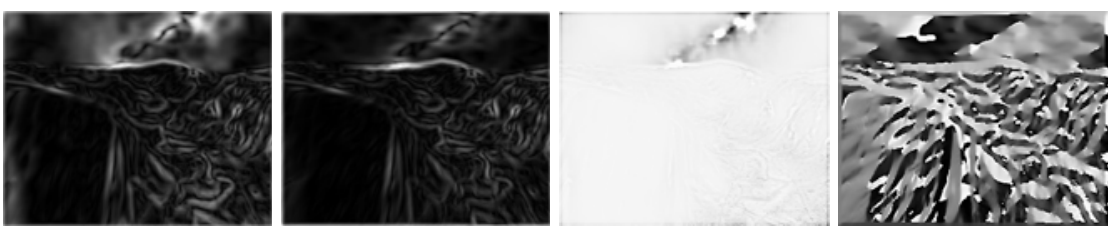

Fig. 5. The 3D conformal signal delivers four local features which can be used for image sequence analysis such as optical flow and motion analysis. From left to right: Curvature, phase and the two parts of the orientation information. 3D convolution mask size $5 \times 5 \times 5$ pixels.

\section{Conclusion}

In this paper a new fundamental idea for locally analyzing multidimensional signals has been presented. The $n$-dimensional domain of the original signal is always limited to its $n$-dimensional Radon space, which restricts the related feature space. To extend the dimension of the related feature space, this problem can be solved by embedding signals in higher dimensional conformal spaces in which the original signal can be analyzed by generalized Hilbert transforms with more degrees of freedom. Without steering and in a rotationally invariant way, local signal features such as amplitude, phase, orientation and curvature can be determined in spatial domain by convolution. The conformal signal can be computed efficiently and can be easily implemented into existing low level image processing steps of Computer Vision applications. Furthermore, exact curvature can be calculated with all the advantages of rotationally invariant local phase based approaches (robustness against brightness and contrast changes) and without the need of any partial derivatives. Hence, lots of numerical problems of partial derivatives on discrete grids can be avoided. All results can be proved mathematically as well as by experiments. Applications of the conformal signal such as object tracking [8] with arbitrary acceleration on three-dimensional data will be part of our future work.

\section{References}

1. Brackx, F., De Knock, B., De Schepper, H.: Generalized multidimensional Hilbert transforms in Clifford analysis. International Journal of Mathematics and Mathematical Sciences (2006) 
2. Felsberg, M.: Low-level image processing with the structure multivector. Technical Report 2016, Kiel University, Department of Computer Science (2002)

3. Felsberg, M., Sommer, G.: The monogenic scale-space: A unifying approach to phase-based image processing in scale-space. Journal of Mathematical Imaging and Vision 21, 5-26 (2004)

4. Grau, V., Becher, H., Noble, J.A.: Phase-based registration of multi-view realtime three-dimensional echocardiographic sequences. In: Larsen, R., Nielsen, M., Sporring, J. (eds.) MICCAI 2006. LNCS, vol. 4190, pp. 612-619. Springer, Heidelberg (2006)

5. Gürlebeck, K., Habetha, K., Sprössig, W.: Funktionentheorie in der Ebene und im Raum. Grundstudium Mathematik. Birkhäuser, Basel (2006)

6. Hahn, S.L.: Hilbert Transforms in Signal Processing. Artech House Inc., Boston (1996)

7. Krause, M., Sommer, G.: A 3D isotropic quadrature filter for motion estimation problems. In: Proc. Visual Communications and Image Processing, Beijing, China, vol. 5960, pp. 1295-1306. The International Society for Optical Engineering, Bellingham (2005)

8. Lichtenauer, J., Hendriks, E.A., Reinders, M.J.T.: Isophote properties as features for object detection. In: CVPR (2), pp. 649-654 (2005)

9. Wietzke, L., Fleischmann, O., Sommer, G.: 2D image analysis by generalized hilbert transforms in conformal space. In: Forsyth, D., Torr, P., Zisserman, A. (eds.) ECCV 2008, Part II. LNCS, vol. 5303, pp. 638-649. Springer, Heidelberg (2008)

10. Wietzke, L., Fleischmann, O., Sommer, G.: Signal analysis by generalized hilbert transforms on the unit sphere. In: Simos, T.E. (ed.) International Conference on Numerical Analysis and Applied Mathematics, AIP Conference Proceedings, Melville, New York, vol. 1048, pp. 706-709 (2008)

11. Wietzke, L., Sommer, G.: The conformal monogenic signal. In: Rigoll, G. (ed.) DAGM 2008. LNCS, vol. 5096, pp. 527-536. Springer, Heidelberg (2008)

12. Wietzke, L., Sommer, G., Schmaltz, C., Weickert, J.: Differential geometry of monogenic signal representations. In: Sommer, G., Klette, R. (eds.) RobVis 2008. LNCS, vol. 4931, pp. 454-465. Springer, Heidelberg (2008) 